\title{
TEM and MultiSlice Simulation Investigation of Si and Ge Nanowires
}

T. Hanrath, D. C. Lee, and B. A. Korgel

Advanced Materials Research Center, University of Texas at Austin

A.C. Diebold

Advanced Materials Research Center, SEMATECH, Austin, TX

Recent TEM characterization of nanowires has shown that silicon and germanium nanowires, synthesized by the supercritical fluid-liquid-solid (SFLS) growth method, have facets and show extra electron diffraction peaks. Additional weaker diffraction spots with hexagonal symmetry at 1/3 (422) spacing are observed in nanowire diffraction on the [111] pole as shown in Figure 1. The nanowire is elongated in the [110] direction. In addition, nanowires often appear to have twinning-type defects in HR-TEM when observed along one direction, but do not show this behavior when rotated as shown in Figure 2. The traditional explanation for this behavior is that different lattice planes diffract when the sample is rotated. This explanation holds true for nanowires also. A multislice simulation was done in order to insure that the facet itself was not the cause of the appearance of facets. This is shown in Figure 3. We will show multi-slice simulations of TEM images of imperfect nanowires were consistent with these observations.

The impact of aberration correction on nanowire imaging is substantial. The aberration corrected HR-TEM image of a $\mathrm{Si}$ nanowire (shown in Figure 4) is free from delocalization. When viewed at high resolution, it shows the crystalline nature of the gold particle that seeds the growth of the nanowire and the epitaxial nature of the first layers of the silicon atoms. Most previous images did not show the crystalline nature of the gold seed particle.

The authors gratefully acknowledge Alex Thesen for the aberration corrected HR TEM image. 


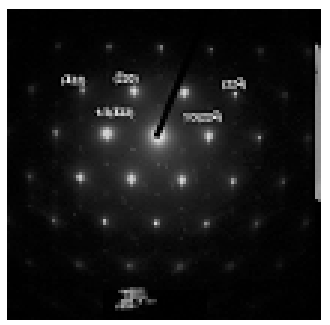

Figure 1. Ge nanowire diffraction pattern on the [111] pole showing the $1 / 3(422)$ reflections
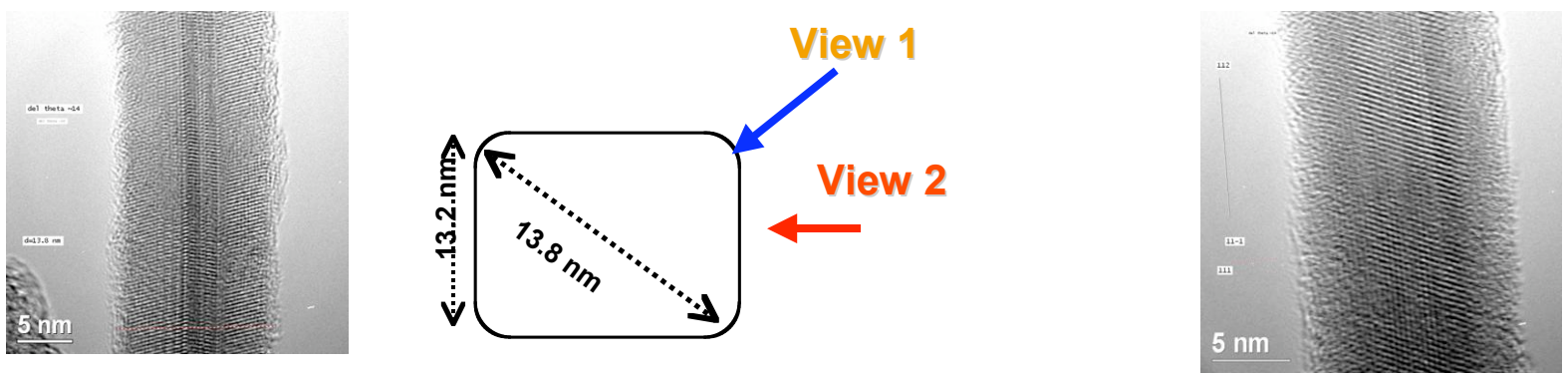

Figure 2. Faceting of a Ge nanowire with [112] growth axis. View 1 on the right shows twinning, while view 2 does not

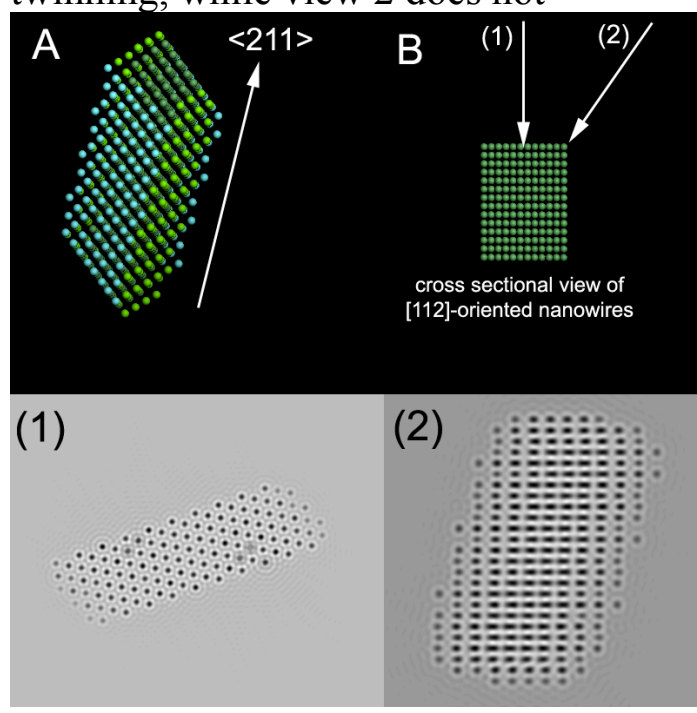

Figure 3. Multislice Simulation showing that facets do not cause twinning to appear in the image

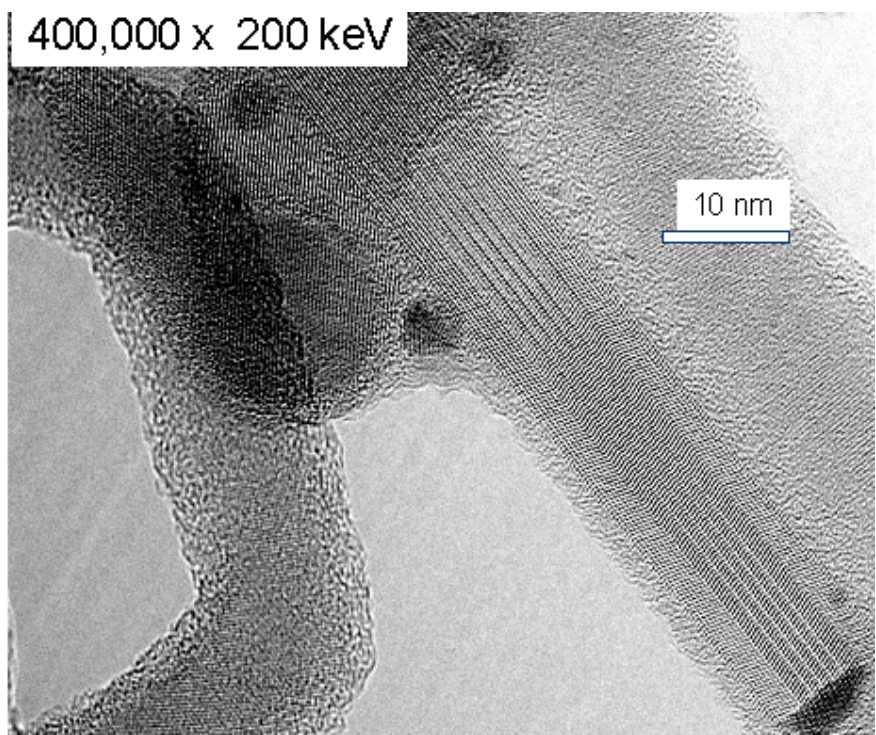

Figure 4. Aberration Corrected HR-TEM image of a Ge nanowire showing twinning and the epitaxial nature of the interface with the gold seed particle 